\title{
Prevalence and Risk Factors of Cigarette Consumption Among the University of Sharjah Students
}

\author{
Ashraf Ahmed Zaghloul ${ }^{1} \&$ Moetaz Elsergany $^{2}$ \\ ${ }^{1}$ Health Services Administration Department, College of Health Sciences, University of Sharjah, Sharjah, UAE \\ ${ }^{2}$ School of Health and Environmental Studies, Hamdan Bin Mohammed Smart University, Dubai, UAE \\ Correspondence: Ashraf Ahmed Zaghloul, Health Services Administration Department, College of Health \\ Sciences, University of Sharjah, Sharjah, UAE. 27272 Sharjah, UAE. Tel: 971-65-057-504. E-mail: \\ azaghloul@sharjah.ac.ae
}

Received: December 10, 2017 Accepted: January 10, 2018 Online Published: January 19, 2018

doi:10.5539/gjhs.v10n3p13

URL: https://doi.org/10.5539/gjhs.v10n3p13

\begin{abstract}
Objective: Smoking is considered a major public health problem throughout the world. Although the burden of a disease attributable to smoking occurs among adults yet, the problem originates in the teenage and adolescence when the majority of smokers have their first experience with cigarettes. The objective of this study was to estimate prevalence of cigarette consumption among the University of Sharjah students.
\end{abstract}

Setting: University of Sharjah, Sharjah campus.

Participants: The total undergraduate student population registered at University of Sharjah (UoS) during the period of study.

Design: A cross-sectional design was followed and included a sample of the University of Sharjah students based on the assumption of a prevalence of $15 \%$ and a degree of precision of $5 \%$ at the $95 \%$ confidence interval for each of the two campuses within the University city (Medical and Health Sciences campus and Non-Medical campus). The designed data collection tool was distributed based on the stratified sampling technique.

Results: The overall prevalence was $28.2 \%$ for both sexes. The prevalence of smoking among males accounted for $44.6 \%$, while the prevalence of smoking accounted for $13 \%$ in females. The highest percentage of type of smoking was cigarettes $52.2 \%$ in males and $78.5 \%$ in females followed by medwakh $30.2 \%$ in males and water-pipe in females $21.5 \%$. A student at a non-medical college, being a non-national, and having parents who were smokers were the common logistic regression predictors of smoking for both sexes in the sample under study.

Conclusion: Tobacco control strategies and preventive measures in the UAE should start as early as preparatory and high school education and be directed towards school students as it seems that the problem is escalating in prevalence and magnitude.

Keywords: smoking, prevalence, risk factor, university, students, cigarette

\section{Introduction}

Smoking is considered a major public health problem throughout the world. It is estimated that smoking and tobacco-induced diseases such as chronic bronchitis, bronchial asthma, and lung cancer lead to approximately 5.4 million deaths annually. By 2030, 8.3 million deaths worldwide will be attributed to these diseases, representing $10 \%$ of deaths globally (Jafari, Haji Zamani, \& Alizadeh, 2011). Although the burden of a disease attributable to smoking occurs among adults yet, the problem originates in the teenage and adolescence when the majority of smokers have their first experience with cigarettes (Polańska, Wojtysiak, Bąk- Romaniszyn, \& Kaleta, 2016).

The epidemic of tobacco use among young people is on the rise globally, in developed and developing countries. According to the Global Youth Tobacco Survey (GYTS) conducted from 1999 to 2009, 21\% of boys and 17\% of girls had smoked cigarettes in the previous 30 days (Öncel, Gebizlioglu, \& Aliev, 2011). The epidemic goes back till 2002 as identified by the WHO Tobacco Atlas, when the rate of male adult smokers was $48 \%$ in Southeast Asia, $43 \%$ in other Asian regions, 38\% in Europe, and 35\% in North America, while that of female adult smokers was $11 \%, 11 \%, 23 \%$, and 21\%, respectively (Takeuchi, Morita, Naito, \& Hamajima, 2010).

Tobacco consumption in males was estimated to be much higher in Arab nations (24.8-61.7\%) compared to 
western nations (19.8-46\%); whereas it was estimated to be lower for females in Arab nations (0.3-7.9\%) compared to western nations (13.7-31.1\%) (Mandil et al., 2010). In many developing countries, the prevalence of smoking among women consistently reported to be lower than in men, where women are traditionally stigmatized for using tobacco (Lopez, Collinshaw, \& Piha, 1994). Eastern Mediterranean Region (EMR) countries, follow the same trend in gender differences in smoking when it comes to conventional methods of tobacco use (cigarette smoking) (Mandil et al., 2010).

The rate of smoking behaviors was found to be high among adolescents with decrease in the age of onset over the time. An issue of great importance among university students, as an educated population which can affect all the strata of society (Jafari et al., 2011).

In a previous study at the University of Sharjah (Mandil, Hussein, Omer, Turki, \& Gaber, 2007), reported that smoking among university students ranged between $13-20 \%$ in males, and $9-11 \%$ in females students. Another study among students at a Saudi university estimated the overall prevalence of smoking at $14.5 \%$, where smoking among male students was (32.7\%), and females (5.9\%) (Mandil et al., 2010). The WHO (World Health Organization, 2015), report for UAE revealed that the prevalence of smoking among the youth for both sexes in the UAE $12.5 \%, 16.5 \%$ among males and $8.4 \%$ among females.

The objective of this study was to estimate prevalence of cigarette consumption among the University of Sharjah (UoS) undergraduate students enrolled during 2016/2017; and to explore associated risk factors which may contribute to cigarette use among both sexes.

\section{Methods}

\subsection{Study Design}

A cross-sectional design was followed for data collection. The study was conducted during the period between March $1^{\text {st }}$ to May10 $0^{\text {th }} 2017$.

\subsection{Sample Size Determination}

Sample size for proportion without replacement was calculated (Equation 1) $(\mathrm{n}=696)$ and a degree of precision of $4 \%$ at the $95 \%$ confidence interval for both campuses (Medical and Non-Medical campuses).

$$
n=\widehat{p}(1-p) \cdot z_{\frac{\alpha}{2}}^{2} / e^{2}
$$

$\mathrm{n}=$ sample size, $\mathrm{p}=$ proportion $\left(0.5\right.$ for smokers and non-smokers, $z_{\frac{\alpha}{2}}^{2}=1.96^{2}, \mathrm{e}=$ degree of precision

\subsection{Sampling}

The total undergraduate student population registered at University of Sharjah (UoS) during the period of study accounted for 11808 students. The inclusion criteria for the sample included full time student status for both genders, and enrolled at the UoS during the academic year 2016/2017. The sample was stratified into two samples from the two campuses in the University. Medical campus students (includes the medical and health sciences colleges) $(\mathrm{n}=2121)$ and Non-Medical campus students (includes all the non-medical colleges, e.g engineering, law, arts, etc; $(\mathrm{n}=9687)$. Further stratification according to gender was followed based on the proportion of male and female students in both campuses. Stratification for the Medical campus yielded a sample of 326 students; where males account for $\mathrm{n}=42$ and females accounted for $\mathrm{n}=284$, and a sample of 370 students for the Non-Medical campus where males accounted for $n=143$ and females accounted for $n=227$. The questionnaire was randomly distributed to the selected samples. Anonymity of participating students was indicated on the cover page of the questionnaire and confidentiality was maintained on all collected questionnaires. The inclusion criteria for the sample were: full time student status (of both genders), enrollment in one of UoS undergraduate programs, during the academic year 2016/2017, with an age range: $17-25$ years.

\subsection{Data Collection Tool}

A questionnaire was developed for data collection based on the Global Adults Tobacco Survey (GATS) (Global Adult tobacco Survey Collaborative Group, 2011). The questionnaire entailed demographic variables as well as possible risk factors including; age (quantitative), gender (male=1, female=2), campus (Medical=1, Non-medical=2), nationality (national $=1$, non-national $=2$ ) are you a smoker? ( $\mathrm{Yes}=1, \mathrm{No}=2$ ), have you ever tried smoking, even just a few puffs? (Yes=1, $\mathrm{No}=2$ ), are you fully aware of the dangers of smoking? (Yes=1, $\mathrm{No}=2$ ), from where did you get your information about smoking? ( $1=$ friends, $2=$ Media e.g; magazine/ newspapers, $3=$ my 
lectures and studies at the university, $4=$ other/ specify), do your parents smoke? $(1=\mathrm{No}, 2=$ father, $3=$ mother, $4=$ both), what is the main reason behind your smoking habit? $(1=$ trying the experience, $2=$ parents habits, $3=$ stress relief, $4=$ friends habits, $5=$ relatives habits), type of smoke ( $1=$ cigarette, $2=$ chewing tobacco, $3=$ pipe, $4=$ =medwakh, $5=$ water-pipe), how often do you smoke? ( $1=$ daily, $2=2-4 /$ week, $3=$ weekly, $4=$ less often), how many cigarettes per day? $(1=1-5,2=6-10,3=10-20,4=20+, 5=$ Not applicable (I don't smoke cigarettes), monthly cost of smoking ( $1=100-200$ AED, $2=200-400$ AED, $3=400-600$ AED, 4=600+AED), Is this considered an economic burden to you? (1=Yes, $2=\mathrm{No})$,

\subsection{Statistical Analysis}

Data were reviewed for completion and accuracy, and analyzed using the Statistical Package for Social Sciences (SPSS) version 21.0. Descriptive statistics as mean, standard deviation and proportion were used to describe the quantitative and qualitative variables. One sample test, and Chi-square tests were used to observe and measure the association between qualitative variables and outcome variables. Binary logistic regression was used to yield risk factors for a dichotomous outcome variable smoking status (Yes/No). Reference categories for the variables included in the equation for both genders were adjusted as follows; age (17 to below 22 years), non-national students, non-medical colleges, awareness of dangers of smoking, parents being smokers. For both crude and adjusted odds ratios, $95 \%$ confidence intervals were calculated. A p-value of $<0.05$ was considered as statistically significant.

\subsection{Ethical Considerations}

This study proposal was granted approval by The Ethics Committee of the Medical Campus at the University of Sharjah on March 2017 (Research Ethics Committee, University of Sharjah, 2017).

\section{Results}

The total number of smokers accounted for 209 students. By using the one sample test for dichotomous variables (smoker/non-smoker), testing the prevalence reported by the WHO (12.5\%), the results yielded a significant difference with a prevalence of $28.2 \%, \mathrm{p}<0.001$ for both sexes in the sample under study. Male smokers accounted for $75.6 \%$ of the total number of smokers, whereas, females accounted for $24.4 \%$. The prevalence of smoking among males accounted for $44.6 \%$, while the prevalence of smoking accounted for $13 \%$ in females.

Table 1 presents the demographic characteristics of the sample under study by smoking habit. The test of significance yielded a significant association between variables gender $\left(X^{2}=90.3\right)$ where the highest proportion of smokers were males $(75.6 \%)$ while the highest proportion of non-smokers were females $(63.2 \%)$, college $\left(\mathrm{X}^{2}=40.5\right)$ where the highest proportion of smokers were enrolled at the non-medical colleges (78.9\%) as well as the highest proportion of non-smokers were also enrolled in non-medical colleges (53.6\%), ever tried smoking (just a few puffs) $\left(X^{2}=280.2\right)$ where the highest proportion of smokers answered (yes, $\left.93.3 \%\right)$ while the highest proportion of non-smokers answered (no, 67.1\%), and parents smoke $\left(\mathrm{X}^{2}=21.3\right)$ where the highest proportion of smokers had smoking parents $(51.2 \%)$ and the highest proportion of non-smokers had non-smoking parents $(67.1 \%)$ respectively.

Table 1. Demographic characteristics by smoking habit (Sharjah, 2017)

\begin{tabular}{|c|c|c|c|c|c|}
\hline & \multicolumn{2}{|c|}{$\begin{array}{l}\text { Smoker } \\
(n=209)\end{array}$} & \multicolumn{2}{|c|}{$\begin{array}{l}\text { Non-Smoker } \\
(\mathrm{n}=532)\end{array}$} & \multirow{2}{*}{$\begin{array}{l}\text { Test of Significance } \\
X^{2}\end{array}$} \\
\hline & No. & $\%$ & No. & $\%$ & \\
\hline \multicolumn{6}{|l|}{ Age } \\
\hline $17-<22$ & 148 & 70.8 & 413 & 77.6 & 3.79 \\
\hline $22+$ & 61 & 29.2 & 119 & 22.4 & \\
\hline \multicolumn{6}{|l|}{ Gender } \\
\hline Male & 158 & 75.6 & 196 & 36.8 & $90.33^{*}$ \\
\hline Female & 51 & 24.4 & 336 & 63.2 & \\
\hline \multicolumn{6}{|l|}{ Nationality } \\
\hline National & 48 & 23.0 & 156 & 29.3 & 3.03 \\
\hline Non-National & 161 & 77.0 & 376 & 70.7 & \\
\hline
\end{tabular}




\begin{tabular}{llllll}
\hline College & 44 & 21.1 & 247 & 46.4 & $40.5^{*}$ \\
Medical College & 165 & 78.9 & 285 & 53.6 & \\
Non-Medical College & 198 & 94.7 & 513 & 96.4 & 1.1 \\
\hline Awareness of dangers of smoking & & 5.3 & 19 & 3.6 & \\
Yes & 11 & & & & \\
No & & & & & \\
\hline Ever tried smoking (just a few & & 93.3 & 135 & 25.4 & $280.2^{*}$ \\
puffs)? & 195 & 6.7 & 397 & 74.6 & \\
Yes & 14 & & & & \\
No & 107 & 51.2 & 175 & 32.9 & $21.3^{*}$ \\
\hline Parents smoke & 102 & 48.8 & 357 & 67.1 & \\
Yes & & & &
\end{tabular}

Table 2 presents the type of smoke by gender. The highest percentage for both genders accounted for cigarettes in males $(52.2 \%)$ and females $(78.5 \%)$. Whereas the second highest percentage in males accounted for midwakh (30.2\% and water-pipe for females (21.5\%). Water-pipe and pipe smoking in males accounted for $6.3 \%$ each.

Table 2. Type of smoke by gender (Sharjah, 2017)

\begin{tabular}{lllllll}
\hline & $\begin{array}{l}\text { Male } \\
(\mathbf{n = 2 0 5})\end{array}$ & & & \multicolumn{2}{l}{$\begin{array}{l}\text { Female } \\
(\mathbf{n}=\mathbf{6 5})\end{array}$} \\
\cline { 2 - 3 } \cline { 5 - 6 } & No. & $\mathbf{\%}$ & & & No. & \% \\
\hline Cigarette & 107 & 52.2 & & 51 & 78.5 \\
Water-pipe & 13 & 6.3 & & 14 & 21.5 \\
Medwakh & 62 & 30.2 & & 0 & 0.0 \\
Pipe & 13 & 6.3 & & 0 & 0.0 \\
Chewing tobacco & 10 & 4.9 & & 0 & 0.0 \\
\hline
\end{tabular}

Table 3 presents the significant logistic regression predictors of smoking in males. The variables namely; college (non-medical) $(\beta)=-1.1, \mathrm{p}<0.0001)$, nationality (non-national) $(\beta)=0.6, \mathrm{p}<0.024)$, and parent is a smoker $(\beta)=-0.6$, $\mathrm{p}<0.007)$

Table 3. Logistic regression predictors in smoking males (Sharjah, 2017)

\begin{tabular}{lllll}
\hline Variable & B & SE & Significance & Exp(B) \\
\hline Constant & 1.07 & 0.4 & & 2.9 \\
College & -1.1 & 0.3 & 0.0001 & 1.9 \\
Nationality & 0.6 & 0.3 & 0.024 & 1.8 \\
Parent smoker & -0.6 & 0.2 & 0.007 & 0.7 \\
\hline
\end{tabular}

$p<0.05$.

Table 4 presents the significant logistic regression predictors of smoking in females. The variables namely; college (non-medical) $(\beta)=-1.1, \mathrm{p}<0.001)$, awareness of danger $(\beta)=1.6, \mathrm{p}<0.04)$, age (17 to below 22 years) $(\beta)=0.96$, $\mathrm{p}<0.02)$ and parent is a smoker $(\beta)=-1.2, \mathrm{p}<0.001)$. 
Table 4. Logistic regression predictors in smoking females (Sharjah, 2017)

\begin{tabular}{lllll}
\hline Variable & B & SE & Significance & Exp(B) \\
\hline Constant & 20.1 & 0.34 & & 0.54 \\
College & -1.1 & 0.35 & 0.001 & 0.31 \\
Awareness of dangers & 1.6 & 0.81 & 0.04 & 4.97 \\
Age (17 - below 22) & 0.96 & 0.4 & 0.02 & 2.63 \\
Parent smoker & -1.2 & 0.4 & 0.001 & 0.29 \\
\hline
\end{tabular}

$p<0.05$.

\section{Discussion}

The study results reveal an alarming rise in the proportion of smokers among university students $(28.2 \%)$ for both sexes compared to a previous study conducted in 2008 (12.5\%) for both sexes (Mandil et al., 2007) and the results based on the data from the National Study of Population Health in the UAE (NSPHUAE) (2007-2009) which accounted for $13.9 \%$ among adolescents between the ages of 13 to 20 years (Barakat-Haddad, Zhang, Siddiqua, \& Dghaim, 2015). A high prevalence of smoking among university students was encountered in other studies, $46.9 \%$ in Greece (Alexopoulos, Jelastopulu, Aronis, \& Dougenis, 2010), 31.6\% in Turkey (Öncel et al., 2011), 31\% in Gaza, Palestine (Abu Shomar, Lubbad, El Ansari, Al-Khatib, \& Alharazin, 2014), and as low as 25.3\% in a university in Tehran (Jafari et al., 2011). According to the American College Health Association, approximately 29\% U.S. college students report lifetime cigarette smoking (Bennett, Deiner, \& Pokhrel, 2017).

Water-pipe smoking continues to be a popular type of tobacco consumption in this region especially for females as previously revealed in other studies (Maziak et al., 2004; WHO, 2006; Badr \& Hamada, 2005) yet, another type of tobacco consumption characteristic for the youth in the UAE which is medwakh came second in prevalence among the sample. The popularity of this type of consumption in this country replaces the water-pipe to third place for males, yet the overall prevalence of medwakh and water-pipe (36.5\%) was closely similar to previous studies in the area $(37.8 \%)$ (WHO, 2006).

Badr and Hamada (2005), also reported that prevalence of smoking among females is on the rise and females are being targeted in the marketing strategies of cigarette industry (Badr \& Hamada, 2005), this was consistent with the high prevalence yielded in the results contrary to our hypothesis of a lower prevalence owing to the customs and cultures in the region which consider such habits unacceptable for females (Maziak et al., 2004; WHO, 2006; Badr \& Hamada, 2005; Al-Mahmoudi \& Amin, 2010).

Affiliated to a non-medical college was a predictor of smoking, but the probability of such a predictor is less than $50 \%$ owing to the negative beta coefficient. Although there is no evidence regarding the type of education and courses taught at university level and its association with smoking, yet there health related course could contribute to the awareness of dangers of smoking and could have affected the results of the sample under study. In the same context, being a multinational university with a diversity of cultures, where the majority of students at the university are non-nationals could have contributed to the nationality variable where non-national being a predictor of smoking.

Living among non-smoking parents is not necessary a preventive factor towards this unhealthy habit, as non-smoking parents of both male and female students were significantly associated with their smoking status in the sample. Within the same context of negative coefficients, the probability is expected to be less than $50 \%$ of the sample owing to the negative beta coefficients yielded from the model. Whereas, contrary to this observation a significant predictive factor for smoking in other studies was having both parents as smokers (Öncel et al., 2011; Barakat-Haddad et al., 2015; Aslam, Zaheer, Rao, \& Shafique, 2014; Geidne, Beckman, Edvardsson, \& Hulldin, 2016; Merdad, Al-Zahrani, \& Farsi, 2007).

Smoke-free environments are protective for young people. They prevent young people from starting to smoke and cause those who do smoke to reduce their smoking (Geidne et al., 2016); therefore more efforts are stringently required for the publics in the UAE towards tobacco control.

\section{Conclusion}

Tobacco control strategies and preventive measures in the UAE should start as early as preparatory and high school education and be directed towards high school students as it seems that the problem is escalating in prevalence and 
magnitude rather than declining. This highlights the importance of supporting anti-tobacco messages and education campaigns targeting families and adolescents regarding the risks of tobacco use. In order to make preventive programs more effective, efforts should be focused on the groups at risk, with a comprehensive approach including multiple factors and involving higher education personnel, parents and the group leaders in tobacco control activities. Projects should be aimed at changing social norms around smoking and providing the youth with knowledge and skills to resist smoking are also needed.

\section{Limitations of the Study}

The results of this study were based on a survey in a major university in United Arab Emirates, and are not necessarily representative for the student population of the entire country. Yet compared with similar studies in university settings, the sample was large and randomly selected; therefore, selection bias is less likely to have occurred. An inherent bias for cross sectional studies lies in bias related to the characteristics of the non-respondents. The conservative cultures and beliefs of Arab students especially females may have influenced the response rate and responses on the data collection tool regarding the sensitive topic of the smoking habit in such societies. Being in an academic and research oriented environment, students are exposed to various exhaustive data collection tools throughout the academic year, thus a possibility of response bias is present in the yielded results.

\section{Conflict of Interest Statement}

The authors declare no conflicts of interest in this work either financial or scientific with any organization. The authors alone are responsible for the content and writing of the paper.

\section{Funding}

This research received no specific grant from any funding agency in the public, commercial or not-for-profit sectors.

\section{References}

Abu Shomar, R. T., Lubbad, I. K., El Ansari, W., Al-Khatib, I. A., \& Alharazin, H. J. (2014). Smoking, awareness of smoking-associated health risks, and knowledge of national tobacco legislation in Gaza, Palestine. Cent Eur J Public Health, 22(2), 80-89. https://doi.org/10.21101/cejph.a4005

Alexopoulos, E. C., Jelastopulu, E., Aronis, K., \& Dougenis, D. (2010). Cigarette smoking among university students in Greece: A comparison between medical and other students. Environ Health Prev Med, 15, 115-120. https://doi.org/10.1007/s12199-009-0110-0

Al-Mahmoudi, H., \& Amin, T. (2010). Pattern and prevalence of smoking among students at King Faisal University, Al-Hassa, Saudi Arabia. East Mediterr Health J, 16(1), 56-64. https://www.ncbi.nlm.nih.gov/pubmed/20214159

Aslam, S. K., Zaheer1, S., Rao1, S., \& Shafique, K. (2014). Prevalence and determinants of susceptibility to cigarette smoking among school students in Pakistan: secondary analysis of Global Youth Tobacco Survey. Substance Abuse Treatment, Prevention, and Policy, 9, 10. https://doi.org/10.1186/1747-597X-9-10

Badr, H., \& Hamada, H. (2005). Stressors and coping strategies of medical students. Gender differences. Saudi Med J, 26(5), 890-2.

Barakat-Haddad, C., Zhang, S., Siddiqua, A., \& Dghaim, R. (2015). Air Quality and Respiratory Health among Adolescents from the United Arab Emirates. Journal of Environmental and Public Health, 284595. https://doi.org/10.1155/2015/284595

Bennett, B. L., Deiner, M., \& Pokhrel, P. (2017). College anti-smoking policies and student smoking behavior: a review of the literature. Tobacco Induced Diseases, 15, 11. https://doi.org/10.1186/s12971-017-0117-z

Geidne, S., Beckman, L., Edvardsson, I., \& Hulldin, J. (2016). Prevalence and risk factors of electronic cigarette use among adolescents: Data from four Swedish municipalities. Nordic Studies on Alcohol and Drugs, (33), 225-240. https://doi.org/10.1515/nsad-2016-0017

Global Adult tobacco Survey Collaborative Group. (2011). Tobacco questions for survey: a subset of key questions from the Global Adult Tobacco Survey (GATS). Atlanta: Centers for Disease Control and Prevention. Retrieved from http://www.cdc.gov/tobacco/global/gats/

Jafari, F., Haji Zamani, A., \& Alizadeh, K. (2011). Reviewing the prevalence of (cigarette) smoking and its related factors in students of Tehran university, Iran. Addict Health. 2011 Summer-Autumn, 3(3-4), 105-10.

Lopez, A. D., Collinshaw, N. E., \& Piha, T. (1994). A descriptive model of the cigarette epidemic in developing 
countries. Tobacco Control, 3, 242-7. https://doi.org/10.1136/tc.3.3.242

Mandil A, BinSaeed A, Al-Dabbagha SAR, Al-Dabbagh, R., Alsaadi, M., \& Khan, M. (2010). Smoking among university students: A gender analysis. Journal of Infection and Public Health, (3), 179-187. https://doi.org/10.1016/j.jiph.2010.10.003

Mandil, A., Hussein, A., Omer, H., Turki, G., \& Gaber, I. (2007). Characteristics and risk factors of tobacco consumption among university of sharjah students, 2005. East Mediterr Health J., 13(6), 1449-1458.

Maziak, W., Fouad, F. M., Asfar, T., Hammal, F., Bachir, E. M., Rastam, S., ...Ward, K. D. (2004). Prevalence and characteristics of narghile smoking among university students in syria. International Journal of Tuberculosis $\&$ Lung Disease the Official Journal of the International Union Against Tuberculosis \& Lung Disease, 8(7), 882-889.

Merdad, L., Al-Zahrani, M., \& Farsi, J. (2007). Smoking habits among Saudi female university students: screening, influencing factors \& risk awareness. Ann Saudi Med, 27, 366-9. https://doi.org/10.1016/j.jiph.2010.10.003

Öncel, S. Y., Ömer, L. G., \& Alioğlu, F. A. (2011). Risk factors for smoking behavior among university students. Turkish Journal of Medical Sciences, 41(6), 1071-1080. https://doi:10.3906/sag-1009-1122

Polańska, K., Wojtysiak, P., Bąkromaniszyn, L., \& Kaleta, D. (2016). Susceptibility to cigarette smoking among secondary and high school students from a socially disadvantaged rural area in poland. Tobacco Induced Diseases, 14(1), 28. https://doi.org/10.1186/s12971-016-0092-9

Research ethics committee. University of Sharjah. (2017).

Takeuchi, Y., Morita, E., Naito, M., \& Hamajima, N. (2010). Smoking rates and attitudes to smoking among medical students: a 2009 survey at the nagoya university school of medicine. Nagoya Journal of Medical Science, 72(72), 151-159.

WHO. (2006). Egyptian Smoking Prevention Research Institute. Tobacco use in shisha: studies on water-pipe smoking in Egypt. Cairo: WHO/EMRO. WHO.

WHO. (2015). Report on the Global Tobacco Epidemic. Report on UAE.

\section{Copyrights}

Copyright for this article is retained by the author(s), with first publication rights granted to the journal.

This is an open-access article distributed under the terms and conditions of the Creative Commons Attribution license (http://creativecommons.org/licenses/by/4.0/). 